\title{
The Effect of Different Filling Methods on the Microleakage of the Tooth Edge after Bulk Resin Filling
}

\author{
Xin Gao*, Jing Di
}

Zhenjiang Stomatological Hospital, Zhenjiang 212001, Jiangsu Province, China

\begin{abstract}
Objective: Analyzing the influence of different filling methods on the microleakage of posterior teeth during the treatment of bulk resin filling. Methods: From January 2019 to January 2020, 68 patients with posterior tooth filling (123 teeth) in our hospital were selected and randomly assigned into three groups (A, B, and C), with 41 teeth in each group. Group A received bulk posterior teeth filling resin treatment, group $B$ received Charisma ${ }^{\circledR}$ resin filling treatment and group $\mathrm{C}$ received FX- II filling treatment. The curative effect and microleakage of posterior teeth between the groups were compared. Results: Comparison of treatment efficiency in group A (97.56\%), group B (85.37\%) and group C (73.17\%) shows that $P<0.05$; comparison of microleakage value and operation time of group A, group B and group $\mathrm{C}$ shows that $P<0.05$. Conclusions: In resin filling treatment, bulk posterior tooth filling resin material should be selected for its high hardness, strong edge adhesion, and natural effects.
\end{abstract}

Keywords: Bulk resin filling; Edge of posterior teeth; Microleakage effect

Publication date: November, 2020

Publication online: 30 November, 2020

"Corresponding author: Xin Gao, 67667106@qq.com

As the dental filling technology continues to develop, resin filling is being gradually applied to posterior tooth restoration, giving a new research direction for dental restoration ${ }^{[1]}$. However, after resin restoration, polymerization induced shrinkage may occur and there may be microleakage between the filling and the tooth, which increases the sensitivity of the tooth and causes secondary dental caries. In severe cases, the filling body may become loose or even fall off ${ }^{[2-3]}$. In recent years, patients have gradually increased their demands for aesthetics, function and repair time after restoration. Dentists often choose large pieces of resin to fill the posterior teeth, which has the advantages of short operation time, low dropout rate, and high hardness. In order to explore the microleakage of the edge of the teeth after the large resin filling treatment, a study was carried out on 68 patients with posterior teeth filling in our hospital. The report is as follows.

\section{Backgrounds and Methods}

\subsection{Background}

From January 2019 to January 2020, 68 patients with posterior dental fillings were selected, with a total of 123 defected teeth, and they were randomly divided into three groups, each with 41 teeth. Among the 68 patients, 36 were males and 32 were females, with an age range of 18-62 years, and the average age was $(41.28 \pm 5.38)$ years old. The samples in this study were not accompanied by systemic diseases and had no history of drug allergy. They all met the diagnostic criteria for caries and did not meet the indications for root canal treatment. The samples were informed of the study and this study is approved by the ethics committee of our hospital. Baseline data analysis of the 68 patients shows $P>0.05$.

\subsection{Treatment Methods}

After the patient is admitted to the hospital, the dental calculus is removed, and the diseased tissue is removed with a slow-speed ball drill to prepare the slope of the cavity edge. Filling materials for Group A and Group B were selected under natural light, followed by acid etching, washing, drying, applying adhesive in sequence, and inserting the forming sheet 
and wedges. Group A was given bulk posterior tooth filling resin (Filtektm Bulk Fill) treatment, one-time filling, polished after $10 \mathrm{~s}$ of illumination; group B was given Charisma ${ }^{\circledR}$ resin (Herae Gusa Dental Co., Ltd.) treatment, filled with layers, polished after $40 \mathrm{~s}$ of illumination. In group $\mathrm{C}$, the formed piece and the wedge were placed to insulate the cavity from moisture, and the affected tooth was partially blow-dried and filled with FX- II material (Nippon Matsukaze). The affected tooth was polished after trimming ${ }^{[4]}$.

\subsection{Assessment of Treatment Effects}

Analysis of the efficiency of posterior tooth filling treatment for patients: after different filling methods, if the fillings did not fall off and no secondary caries were found, it is considered to be Markedly Effective; if the fillings fell off slightly but no secondary caries occurred, and the occlusal function recovered after the second filling, it is considered to be effective; if the filling material is completely off, and the occurrence of secondary caries is found, it is considered to be Ineffective.
Analysis of the microleakage value of the posterior tooth edge of patients with filling treatment: A stereo microscope at 40x magnification was used to measure, and a vernier caliper was used to give accuracy down to $0.1 \mathrm{~mm}$, the average of two readings of the unified profile was used as the final value ${ }^{[5]}$.

\subsection{Statistical Study}

In this paper, the data of posterior teeth filling treated patients were analyzed by SPSS 33.0 , where $\%$ is the statistical method of count data, $\pm \mathrm{s}$ is the statistical method of measurement data, and X2 and t tests were used to check the differences in data. $P<0.05$ indicates significance for comparison.

\section{Results}

\subsection{Assessment on the Treatment Effects in the Three Groups}

After bulk posterior tooth resin filling treatment, the treatment efficiency in group A was $97.56 \%$, group B $85.37 \%$ and group C $73.17 \%$. Significant differences were found between the groups, $P<0.05$. See Table 1 .

Table 1. Comparison of Treatment Efficiency in Patients with Posterior Tooth Filling $(n, \%)$

\begin{tabular}{cccc}
\hline Group & Markedly Effective & Effective & Ineffective \\
\hline A $(n=41)$ & $31(75.61)$ & $9(21.91)$ & $1(2.44)$ \\
B $(n=41)$ & $28(68.29)$ & $7(17.07)$ & $6(14.63)$ \\
C $(n=41)$ & $24(77.42)$ & $6(14.63)$ & $11(26.83)$ \\
$\chi^{2}$ & - & - & - \\
$P$ & - & - & - \\
\hline
\end{tabular}

2.2 Comparison of Microleakage Value and Operation Time between the Three Groups

After bulk posterior tooth resin filling treatment, the microleakage value and operation time of group A were found to be superior over both group B and group $\mathrm{C}, P<0.05$.See Table 2 .

Table 2. Comparison of Microleakage Value and Operation Time after Posterior Tooth Filling Treatment $(\bar{x} \pm s)$

\begin{tabular}{ccc}
\hline Group & Microleakage Value (mm) & Operation Time (s) \\
\hline $\mathrm{A}(n=41)$ & $0.62 \pm 0.15$ & $180.38 \pm 12.75$ \\
$\mathrm{~B}(n \mathrm{n}=41)$ & $0.81 \pm 0.26$ & $276.84 \pm 16.29$ \\
$\mathrm{C}(n \mathrm{n}=41)$ & $0.97 \pm 0.35$ & $342.28 \pm 17.16$ \\
$t$ & $4.0531 / 5.8854$ & $29.8576 / 48.4916$ \\
$P$ & $<0.05$ & $<0.05$ \\
\hline
\end{tabular}

\section{Discussions}

With the wide application of resin filling materials, more options are available for the treatment of posterior teeth filling. Resin filling therapy has the following advantages: less invasive tooth preparation, restoration of the natural color of the tooth, and ability to fully integrate the dentin. However, the resin filling treatment has a certain irritation to the dental pulp, and there is a characteristic of polymerization induced contraction. Some patients have a higher risk of microleakage after resin filling treatment ${ }^{[6-7]}$. Microleakage is the presence of microscopic gaps between the tooth and the restoration material, which is permeable to bacteria, ions, molecules and liquids. After microleakage occurs, the sensitivity 
of the tooth can increase and the filling body can become discolored, causing secondary tooth decay, pulpitis or apical periodontitis, resulting in possible loosening and falling off of the filling ${ }^{[8]}$. Combining the analysis of clinical practice, the microleakage at the edge of the posterior teeth may be related to the following factors: filling material properties, such as matrix performance and inorganic filler content and etc.; clinical factors, such as filling method, cavity type, light and temperature. In order to reduce the aforementioned risk of polymerization induced shrinkage, traditional resin filling adopts layered filling technology treatment, and the filling thickness of each layer does not exceed $2 \mathrm{~mm}$, which reduces the risk of microleakage and improves the curing rate and success rate ${ }^{[9]}$. However, the layered filling operation is more difficult and the operation time is longer, resulting in lower patient acceptance. The moisture insulating effect is limited, which affects the efficiency to a certain extent. In recent years, the bulk resin filling technology has gradually matured, which can reduce operating steps, has low technical requirements, short operating time, low polymerization shrinkage, and high patient satisfaction. In this study, a bulk posterior tooth filling resin material with good physical properties was selected, which effectively reduces the risk of microleakage at the edge of the posterior tooth and the risk of discoloration of the filling material.

Bulk posterior tooth filling resin is a new type of resin filling material. It can fill $4 \mathrm{~mm}$ at a time, reducing the shrinkage stress during the polymerization process and avoid the risk of secondary dental caries after the operation. The filling is uniform during the operation and can be fitted to the cavity wall. The illumination time is only $10 \mathrm{~s}$ and the transparency is close to tooth enamel, which can restore the aesthetics of teeth to the greatest extent ${ }^{[5]}$. In addition, the bulk resin filling material is less affected by light, has sufficient clinical shaping time, good wettability, can reach narrow areas, seals the dentin tubules, and promotes the full integration of the teeth and the filling ${ }^{[10]}$. Combined with this study, the treatment efficiency, microleakage value and operation time of group A are all better than those of group $\mathrm{B}$ and $\mathrm{C}(P<0.05)$, indicating that the bulk resin filling treatment has better sealing performance.

In conclusion, in the filling treatment of posterior teeth, the bulk filling resin has high abrasion resistance, good sealing performance, safety and feasibility, and is worthy of promotion.

\section{References}

[1] Liang Zhao. Clinical analysis of composite resin filling in the treatment of posterior tooth defects [J]. The Journal of Medical Theory and Practice, 2019, 032(024):4028-4029.

[2] Hui Min Wang, Zeng Bo Zhao, Zhi Yu Chen, et al. The influence of different methods and filling materials on the microleakage of deciduous teeth [J]. Journal of Modern Stomatology, 2020, 034(003):169-171.

[3] Mulgaonkar A, Ataide I N D, Fernandes M, et al. Effect of bromelain enzyme on the microleakage of composite resin restorations after external tooth bleaching: An in vitro study[J]. Journal of Conservative Dentistry, 2019, 22(5):436.

[4] Yun Qing Zhang, Zhi Xiong Yang, Chang Ming Liu. Clinical Value Analysis of Bulk Resin for Filling Primary Molars after Root Canal Treatment [J]. The Department of Oral Medicine Electronic Magazine, 2020, 007(001):53-55.

[5] Nian Hua Chen. Analysis on the effect of different filling materials in the treatment of posterior dental caries $[\mathrm{J}]$. The Department of Oral Medicine Electronic Magazine, 2020, 007(003):143,151.

[6] Lei Zhang, Pei Lin Cao, Yi Sha Pan, et al. The effect of different bulk filling resins on the adhesion of class II cavity gingival wall [J]. Journal of Oral Science Research, 2018, 34(03):319-322.

[7] Chao Zhang, Bin Yang, Yong Wang. Preliminary study on the suitability of the new self-curing resin for filling the surface edge and internal interface of class $\mathrm{V}$ cavities [J]. Chinese Journal of Geriatric Dentistry, 2020, 55(03):171-177.

[8] Chao Zhang, Bin Yang, Yong Wang. Preliminary study on the suitability of the new self-curing resin for filling the surface edge and internal interface of class $\mathrm{V}$ cavities [J]. Chinese Journal of Geriatric Dentistry, 2020, 055(003):171-177.

[9] Ling Zhang, Lin Lin Zhu, Jing Bi. Comparative Study on Edge Microleakage of Different Photocuring Filling Materials [J]. Chinese Journal of Practical Stomatology, 2018, 11(3): 176178.

[10] Mei Yi Du, Hong Wang, Yuan Ning Liu, et al. Study on the bonding and flexural strength of Sonicfill system and its influence on the edge adhesion of posterior teeth $[\mathrm{J}]$. Chinese Journal of Geriatric Dentistry, 2020, 018(001):30-34. 